ARTIGO

\title{
ESTUDOS SOBRE A CAPACIDADE DE HOSPEDAGEM DE GERAÇÃO DISTRIBUIIDA EM SISTEMAS ELÉTRICOS DE POTÊNCIA UTILIZANDO ALGORITMO GENÉTICO ${ }^{1}$
}

\author{
Renan Souza Moura ${ }^{2}$ \\ Carine Flávia dos Reis \\ Rarena Kellen Reis Pedrosa \\ Thuanny Reis Neves
}

\begin{abstract}
RESUMO
Há um crescente interesse em inserir fontes de energias renováveis nos sistemas elétricos de potência. Para isso, é preciso conhecer a potência máxima que pode ser instalada em um sistema sem causar problemas operacionais. Esta potência máxima é definida como a capacidade de hospedagem de geração distribuída do sistema. Para analisar quais os lugares mais apropriados para inserir estas fontes, a técnica do vetor tangente foi utilizada, enquanto os valores de potência foram calculados por meio da implementação de um algoritmo genético, que buscou as melhores soluções, dentro de um conjunto, através de técnicas evolutivas. Para analisar se um indivíduo é mais bem condicionado que outro, a curva de Potência ativa versus Tensão (curva PV), responsável por indicar o máximo carregamento de um sistema elétrico, foi utilizada como função objetivo. A metodologia foi experimentada em dois sistemas-teste do Instituto de Engenheiros Eletricistas e Eletrônicos (IEEE) e em um sistema real. Em todas as simulações, consideraram-se os limites operacionais dos sistemas analisados.
\end{abstract}

Palavras-chave: Sistema elétrico. Capacidade de hospedagem. Algoritmo genético. Curva PV.

\section{INTRODUÇÃO}

Com o crescente aumento na demanda de energia do sistema elétrico de potência, é indispensável garantir que ele opere dentro dos limites de estabilidade. Sendo assim, é imprescindível analisar continuamente o quão distante o sistema trabalha desses limites operacionais. Dentro desse contexto, há grande interesse acadêmico, e também por parte das concessionárias, em realizar essas análises, para garantir que o sistema permaneça estável, evitando possíveis danos (AFFONSO; DA SILVA; DA SILVA, 2007; FERREIRA, 2011).

O sistema elétrico de potência deixa de ser estável quando perturbações causam colapsos na rede, ocasionando uma queda de tensão irreversível (KUDUR et al. 2004;

\footnotetext{
${ }^{1}$ Como citar este artigo: MOURA, Renan Souza et al. Estudos sobre a capacidade de hospedagem de geração distribuída em sistemas elétricos de potência utilizando algoritmo genético. ForScience: revista científica do IFMG, Formiga, v. 7, n. 1, e00452, jan./jun. 2019. DOI: 10.29069/forscience.2019v7n1.e452.
}

\footnotetext{
${ }^{2}$ Autor para correspondência: Renan Souza Moura. E-mail: renan.moura@ifmg.edu.br.
} 
MOURA, R. S. et al. Estudos sobre a capacidade de hospedagem de geração distribuída em sistemas 2 elétricos de potência utilizando algoritmo genético

SOUZA; QUINTANA, 1993). Isso pode causar inúmeros prejuízos, que podem ser financeiros, ou problemas permanentes no sistema elétrico, causando desconforto à população.

Uma importante ferramenta é a análise do fluxo de potência de um sistema, que permite conhecer as condições de operação de um sistema elétrico de energia em regime permanente. Para isso, é preciso levar em consideração as características do sistema, como número de barramentos, transformadores, geradores, cargas e capacitores presentes nele. A partir disso, são realizadas análises computacionais, compostas por equações não lineares. Um método muito utilizado para a realização dessas soluções é o método de Newton-Raphson, que permite a resolução de equações complexas e possui uma boa precisão e convergência do algoritmo (CANOSSA, 2007).

Ao aplicar o método de Newton-Raphson para a análise do fluxo de potência de um sistema, é necessário conhecer a magnitude e o ângulo de fase da tensão em cada um dos seus barramentos. Assim, pode-se determinar a distribuição do fluxo de potência ativa e reativa em um sistema de transmissão (LODDI, 2010).

Por meio da análise do fluxo de potência de um sistema, é possível realizar a construção de suas curvas PVs, as quais são obtidas ao simular sucessivos fluxos de potência, aumentando gradativamente a carga do sistema, relacionando tensão e potência em cada barramento. A partir da curva PV de um determinado barramento, pode-se prever a máxima potência que pode ser suprida a uma determinada carga. Durante o cálculo da curva PV, o aumento da carga do sistema é acompanhado de uma atenuação no nível de tensão. Assim, à medida que a tensão no sistema diminui, chegará um ponto em que o sistema deixará de ser estável, podendo causar problemas de colapso de tensão. Esse ponto é definido como o ponto de máximo carregamento do sistema e está associado à bifurcação sela-nó (ALVES, 2000; ALMEIDA et al., 2011; GUIMARÃES et al., 2011).

Estudos relacionados com a construção da curva PV têm sido fundamentais no que diz respeito à estabilidade de tensão de pequenas perturbações, que são aquelas em que suas variáveis de estado não sofrem muita alteração ao tentar encontrar um novo ponto de equilíbrio (ALMEIDA et al., 2013).

A inserção de fontes renováveis no sistema elétrico de potência tem sido um assunto cada vez mais debatido. Entretanto, assim como qualquer fonte, deve existir um valor limite de potência que pode ser instalado no sistema sem causar perturbações na rede (SANTOS; ISSA, 2014; DE OLIVEIRA, 2015; SANTOS et al., 2015). Este limite é definido como a 
capacidade de hospedagem e deve ser considerado quando se deseja ampliar a quantidade de carga abastecida pelo sistema indicado pela curva PV.

Para analisar os lugares mais apropriados para inserir estas fontes no sistema, de forma a ampliar a margem de máximo carregamento indicada pela curva PV, foi utilizada a técnica do vetor tangente (SOUZA; QUINTANA, 1993).

A técnica do vetor tangente permite identificar o barramento crítico de um sistema analisado. Entende-se como barramento crítico aquele que apresenta maior variação do módulo da sua tensão durante a obtenção da curva PV. Uma vez definida a área que apresenta menor suporte de potência reativa, área formada pelo barramento crítico e a sua vizinhança, o algoritmo genético insere injeções de potência nestas barras e utiliza a curva PV como função objetivo.

O algoritmo genético é um método heurístico de otimização global. Ele consiste em uma técnica evolutiva, que tem por objetivo encontrar a melhor solução para um problema utilizando conceitos de genética básica. Populações de indivíduos são criadas e submetidas a operadores genéticos, tais como: seleção - busca indivíduos mais aptos para a solução do problema; recombinação - realiza o cruzamento entre dois indivíduos para se obter novos indivíduos; e mutação - modifica a estrutura um indivíduo selecionado aleatoriamente para a obtenção de novas opções (LINDEN, 2012).

A utilização do algoritmo genético como solução do problema justifica-se por sua generalização, ou seja, não necessita de informação da estrutura da função a ser otimizada. Entre suas principais vantagens, estão: a forma como a técnica trabalha, utilizando parâmetros codificados, e não os parâmetros propriamente ditos; o fato de partir de uma população de soluções alternativas, e não de uma única solução, ao realizar as simulações; e a utilização de regras de transição probabilísticas, e não determinísticas (KAGAN et al., 2009).

\section{OBJETIVO}

O principal objetivo do trabalho foi propor um método de otimização da capacidade de hospedagem de geração distribuída de sistemas elétricos de potência baseado em algoritmo genético. 


\section{REFERENCIAL TEÓRICO}

\subsection{Fluxo de potência}

O cálculo de fluxo de potência permite determinar as condições operativas de um sistema elétrico de potência em regime permanente (MONTICELLI, 1983). Devido à não linearidade das equações que descrevem um sistema elétrico de potência, este trabalho utilizou o método iterativo de Newton-Raphson, onde a seguinte analogia matemática elétrica, indicada pela Tabela 1, é considerada:

Tabela 1 - Analogia matemática elétrica do processo de fluxo de potência

\begin{tabular}{cc} 
Matemático & Físico \\
\hline $\mathrm{f}(\mathrm{x})=0$ & $\sum \mathrm{P}=0$ \\
$\mathrm{x}$ & $\sum \mathrm{Q}=0$ \\
$\mathrm{~V}$ e $\Theta$ & (variáveis de estado) \\
(variável de estado) & Aceitar uma tolerância, neste caso, equivale a aceitar \\
diferença entre duas soluções for menor que uma & uma pequena violação na lei de Kirchoff \\
tolerância $\varepsilon$ &
\end{tabular}

Fonte: Moura e Eto (2007).

Onde $\sum P=0$ e $\sum Q=0$ são as equações de potências injetadas ativas e reativas do cálculo de fluxo de potência, respectivamente.

\subsection{Curva PV}

O método empregado para se obter curvas PVs se dá por sucessivas soluções do fluxo de potência. A cada nova iteração, realiza-se o incremento de carga no sistema elétrico, a fim de obter a tensão em cada um dos barramentos do sistema. 
Por meio dessa curva (Figura 1), é possível relacionar a tensão em um determinado barramento de acordo com o aumento de sua carga. Assim, pode-se determinar o ponto de máximo carregamento, que é a máxima carga que pode ser inserida nesse barramento. A distância entre o ponto operacional e o máximo carregamento é a margem de carga desse sistema (MOURA, 2016).

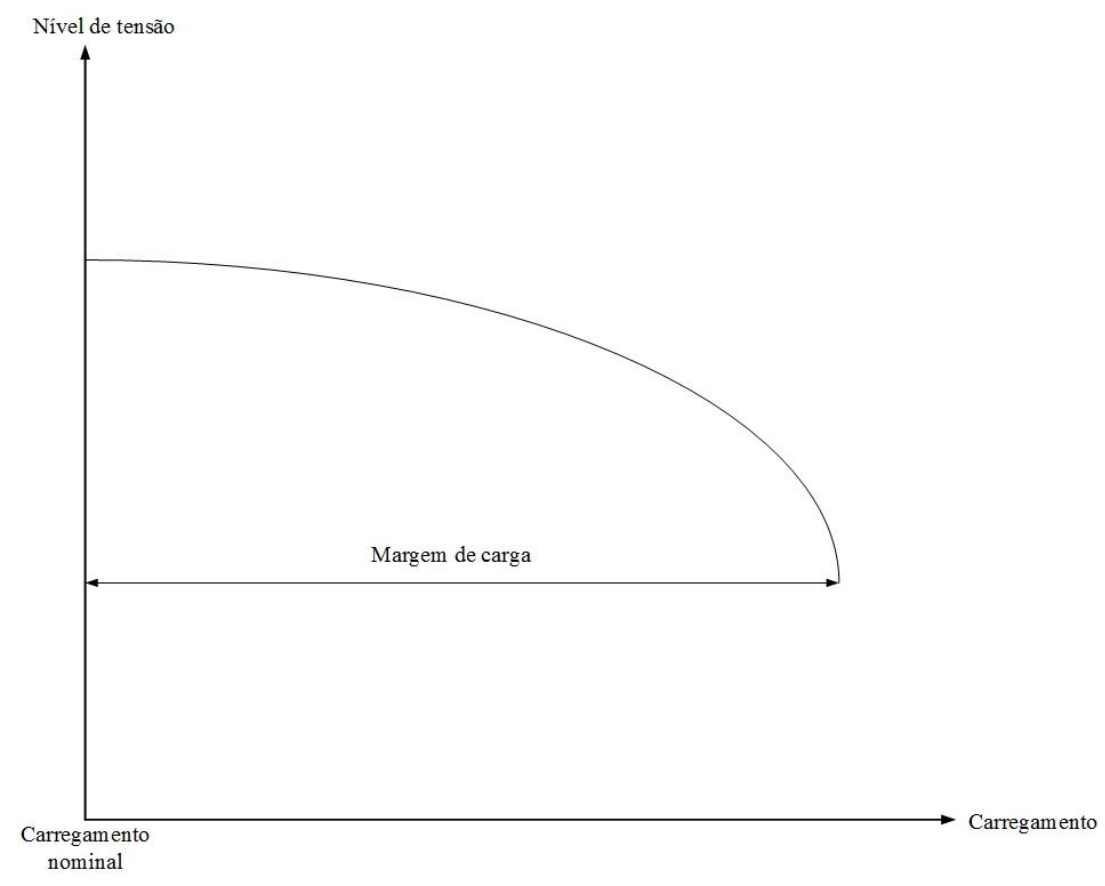

Figura 1- Modelo de Curva PV

Fonte: Autores.

As margens de carga, para dois sistemas-teste do IEEE e o Sistema Sul Sudeste brasileiro, são apresentadas na Tabela 2 .

Tabela 2 - Ponto de máximo carregamento dos sistemas-teste do IEEE

\begin{tabular}{cc} 
Sistemas & Margem de carga [pu] \\
\hline $\mathbf{1 4}$ barras & 1.93 \\
$\mathbf{3 0}$ barras & 1.55 \\
Sistema Sul Sudeste & 1.11 \\
\hline
\end{tabular}

Fonte: Autores.

Os valores das margens de carregamento dos sistemas indicados na Tabela 2 são próximos aos obtidos em (MOURA, 2016). Diferentes valores adotados de tolerância no 
MOURA, R. S. et al. Estudos sobre a capacidade de hospedagem de geração distribuída em sistemas 6 elétricos de potência utilizando algoritmo genético

cálculo de fluxo de potência e no passo de aumento de carregamento podem ser considerados motivos para explicar a pequena discrepância de valores entre a Tabela 2 (MOURA, 2016).

Para ampliar a margem de carga desses sistemas, utilizou-se o algoritmo genético.

\subsection{Algoritmo Genético}

A primeira etapa para a elaboração do algoritmo genético é determinar a função objetivo em questão. No caso deste trabalho, a função objetivo foi a maximização da margem de carga da curva PV.

Feito isto, é realizada a seleção dos indivíduos da primeira geração do algoritmo, determinada de maneira aleatória. Assim, os indivíduos mais aptos dessa primeira geração são escolhidos para efetuarem cruzamento por meio da "Roleta Viciada", onde os indivíduos com maior "peso", ou seja, com chances de melhor solução, terão maior probabilidade de serem escolhidos, e os de menor peso terão, consequentemente, menor chance. A roleta seleciona esses indivíduos tendo como função a margem da curva PV. Assim, podemos dizer que, inicialmente, o algoritmo tem uma natureza aleatória, mas, depois, a própria seleção natural (cruzamento e mutação) faz o trabalho de escolher os mais aptos.

No problema em questão, os indivíduos foram formados pelas potências ativas e reativas geradas por todos os barramentos pertencentes à área crítica. As áreas críticas, presentes na Tabela 3, foram calculadas por meio da técnica do vetor tangente (SOUZA; QUINTANA, 1993), que permite determinar quais barramentos apresentam maior variação de tensão e, consequentemente, necessitam de maior suporte de potência reativa, durante a obtenção da curva PV. A Figura 2 exemplifica a constituição de um indivíduo pertencente ao sistema sul sudeste brasileiro.

Tabela 3- Áreas críticas obtidas pelo método do vetor tangente

\begin{tabular}{cc}
\hline Sistemas & $\begin{array}{c}\text { Barras pertencentes à } \\
\text { área crítica }\end{array}$ \\
\hline 14 barras & $4,6,7,9,10,12,13,14$ \\
30 barras & $25,27,28,29,30$ \\
Sistema Sul Sudeste & $6,8,63,64$ \\
\hline
\end{tabular}

Fonte: Autores. 
MOURA, R. S. et al. Estudos sobre a capacidade de hospedagem de geração distribuída em sistemas 7 elétricos de potência utilizando algoritmo genético

Indivíduo

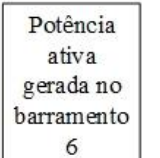

6
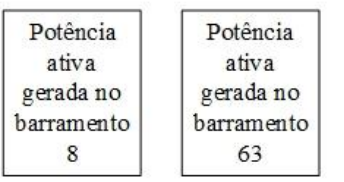

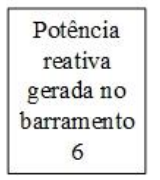

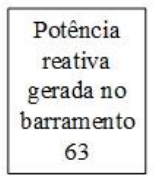

Potência
reativa
gerada no
barramento
64

Figura 2- Constituição de um indivíduo pertencente ao Sistema Sul Sudeste Fonte: Autores.

O número de indivíduos de cada população é igual à quantidade de barramentos do sistema analisado. Por exemplo, as populações dos sistemas de 14 e de 30 barramentos apresentam 14 e 30 indivíduos, respectivamente, em cada geração. Durante a evolução das populações, a quantidade de indivíduos é mantida fixa. A única exceção é a quantidade de indivíduos do sistema sul sudeste, que apresenta 65 barramentos, onde foi adotada uma população com 66 indivíduos, pois, durante o cruzamento dois a dois, outros dois indivíduos são gerados, os quais farão parte da próxima geração. A Figura 3 ilustra o processo de cruzamento empregado neste trabalho para o sistema sul sudeste.

$\mathrm{Na}$ fase de cruzamento, é levada em conta a possibilidade de mutação. Neste estudo, a mutação considerada foi de 1\%. Para implementá-la, é sorteado um número entre 0 e 99 caso o número zero for o selecionado, o indivíduo sofrerá mutação em um de seus genes; do contrário, o indivíduo permanece sem nenhuma alteração.

Este processo se repete até se obter o número de gerações desejadas. No algoritmo implementado, optou-se por realizar 50 gerações, sendo que, em cada uma delas, identificouse o melhor indivíduo para atender à função objetivo. Ao final das 50 gerações, a melhor solução é a melhor entre as 50 melhores soluções armazenadas (técnica do elitismo). 
MOURA, R. S. et al. Estudos sobre a capacidade de hospedagem de geração distribuída em sistemas 8 elétricos de potência utilizando algoritmo genético

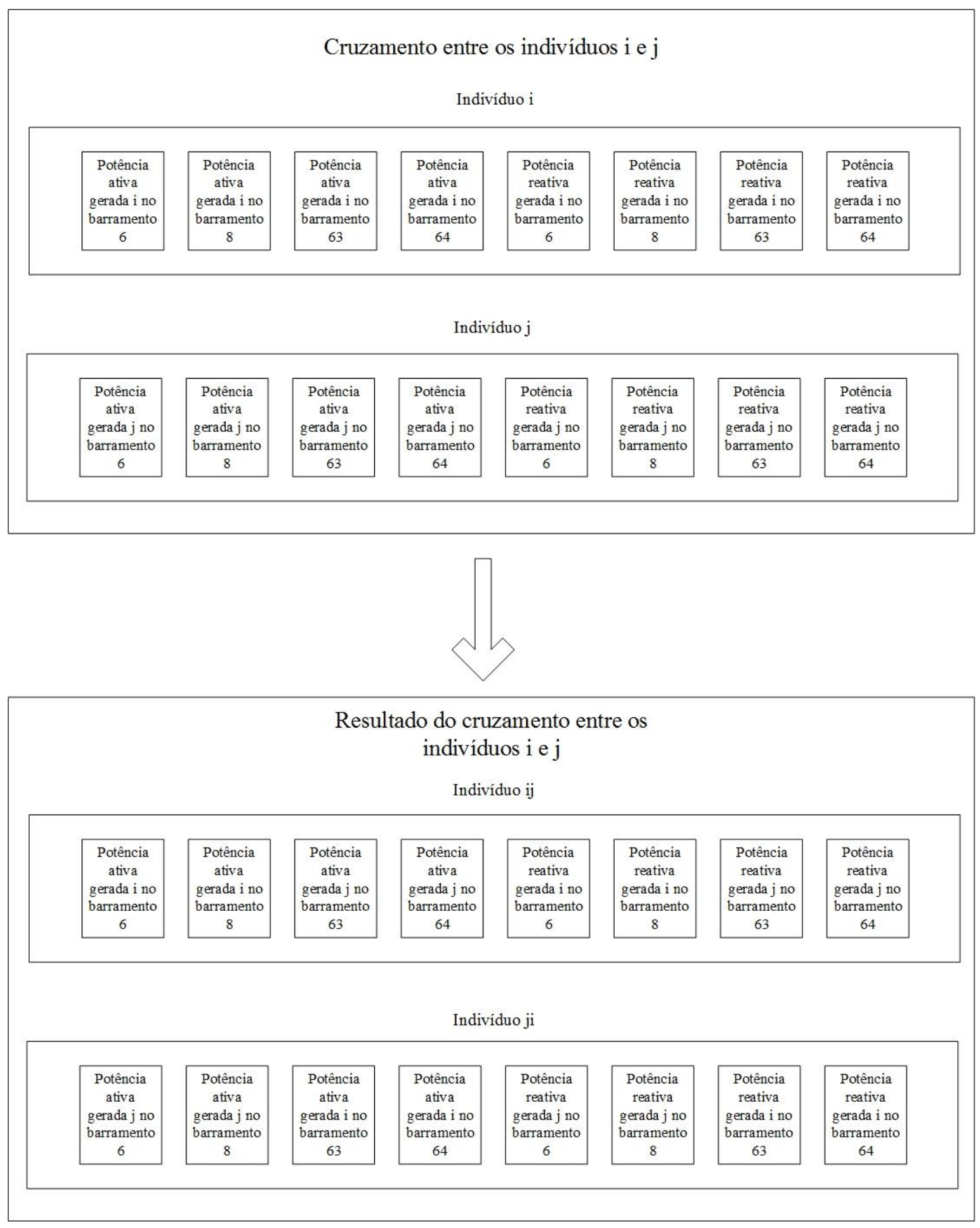

Figura 3 - Cruzamento entre indivíduos pertencentes ao sistema sul sudeste Fonte: Autores. 
MOURA, R. S. et al. Estudos sobre a capacidade de hospedagem de geração distribuída em sistemas 9 elétricos de potência utilizando algoritmo genético

\section{RESULTADOS DE SIMULAÇÃO}

Como o algoritmo genético apresenta uma população inicial escolhida de maneira randômica, todas as respostas das simulações desta seção foram repetidas diversas vezes e apresentaram os mesmos resultados.

Ao utilizar a técnica de busca do algoritmo genético para calcular a capacidade de hospedagem que permite o aumento da margem de carga, observaram-se os seguintes valores de margem de carregamento dos sistemas indicados pela Tabela 4. As Figuras 3, 4 e 5 representam as curvas PVs após a aplicação do algoritmo genético.

Tabela 4 - Novos valores de máximo carregamento dos sistemas-teste do IEEE utilizando algoritmo genético

\begin{tabular}{cccc} 
Sistemas & Barras críticas & $\begin{array}{c}\text { Margem de } \\
\text { carga [pu] }\end{array}$ & $\begin{array}{c}\text { Aumento da } \\
\text { margem [\%] }\end{array}$ \\
\hline 14 barras & $4,6,7,9,10,12,13,14$ & 3,3540 & 73,78 \\
30 barras & $25,27,28,29,30$ & 1,6640 & 7,36 \\
Sul Sudeste & $6,8,63,64$ & 1,1850 & 6,76 \\
\hline
\end{tabular}

Fonte: Autores.

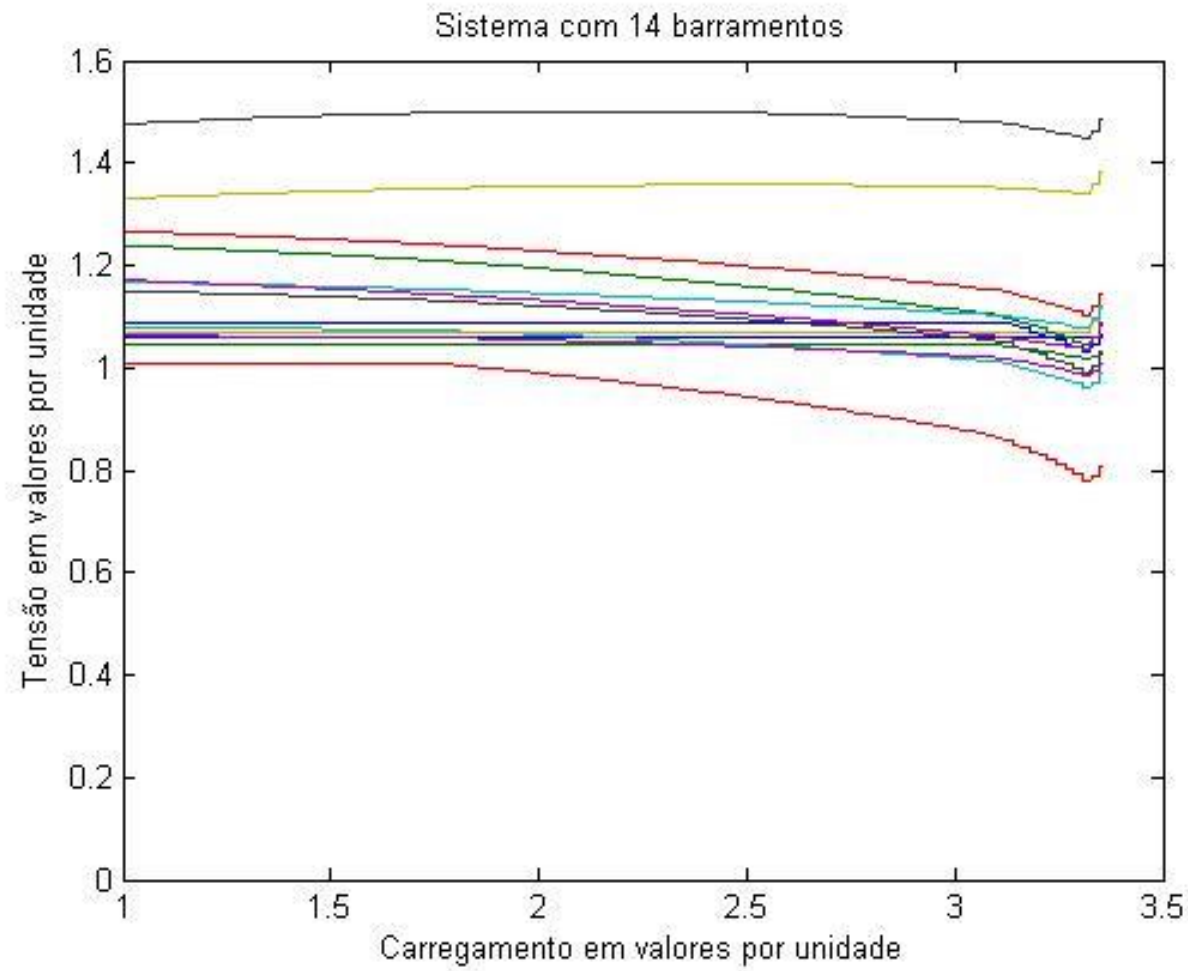

Figura 4 - Curva PV para o sistema com 14 barramentos após a aplicação do algoritmo genético Fonte: Autores. 
MOURA, R. S. et al. Estudos sobre a capacidade de hospedagem de geração distribuída em sistemas 10 elétricos de potência utilizando algoritmo genético

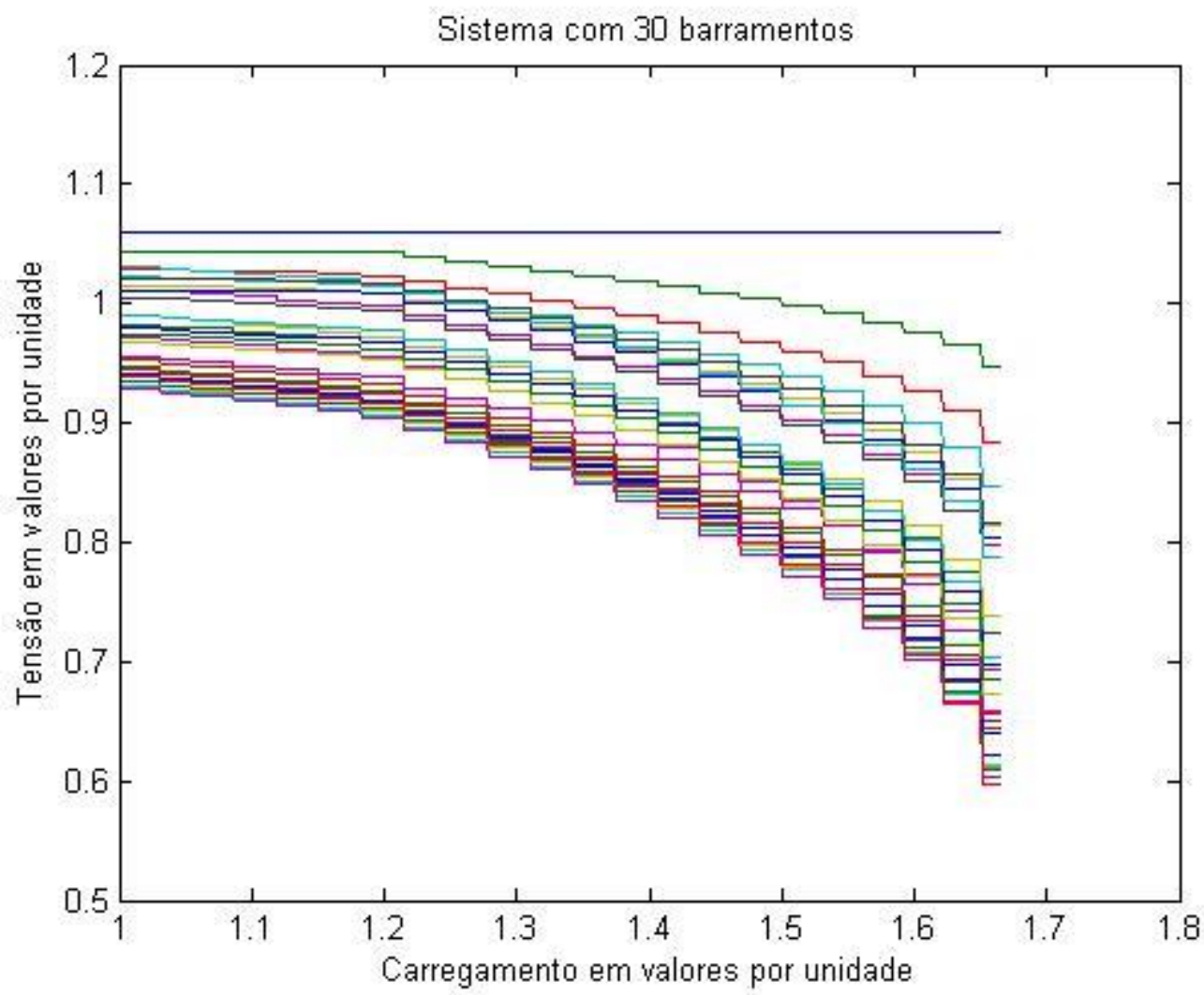

Figura 5- Curva PV para o sistema com 30 barramentos após a aplicação do algoritmo genético Fonte: Autores.

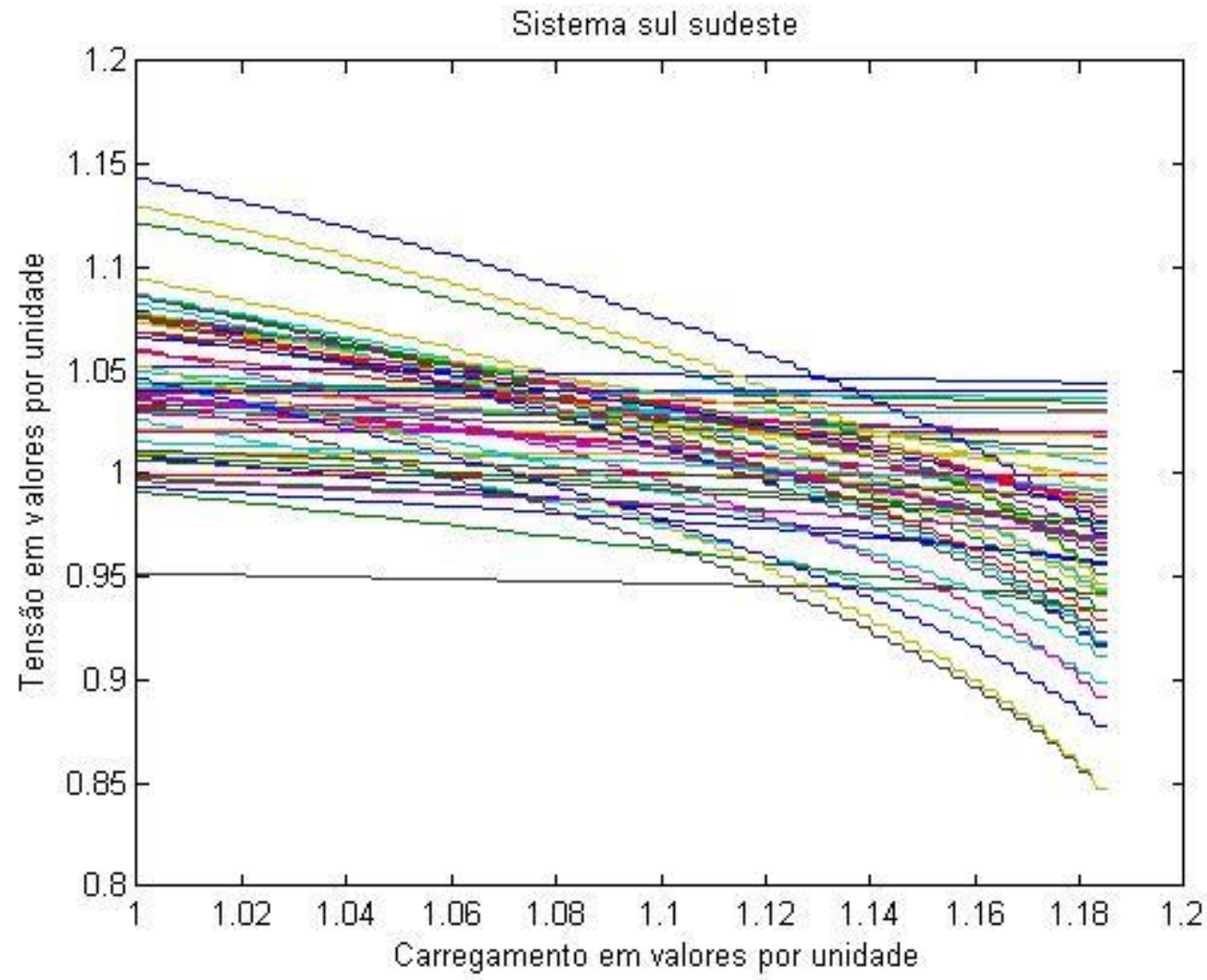

Figura 6 - Curva PV para o sistema sul sudeste após a aplicação do algoritmo genético Fonte: Autores. 
As potências geradas ativas e reativas, encontradas pela técnica de busca do algoritmo genético, são mostradas nas Tabelas 5 e 6, respectivamente. Os valores de potência destas Tabelas, quando combinados na inicialização do cálculo da curva PV, permitem o aumento de carga indicado pela Tabela 4 .

Tabela 5 - Potências ativas geradas nas barras críticas

\begin{tabular}{|c|c|c|}
\hline Sistemas & Barras críticas & $\begin{array}{l}\text { Potências ativas nas barras } \\
\text { críticas (p.u. na base de } 100 \text { MVA) }\end{array}$ \\
\hline 14 barras & $4,6,7,9,10,12,13,14$ & $\begin{array}{l}P_{\mathrm{G} 4}=-0,1506 \\
P_{\mathrm{G} 6}=-0,6987 \\
\mathrm{P}_{\mathrm{G} 7}=0,8328 \\
P_{\mathrm{G} 9}=-0,3308 \\
\mathrm{P}_{\mathrm{G} 10}=0,1283 \\
\mathrm{P}_{\mathrm{G} 12}=-0,3606 \\
\mathrm{P}_{\mathrm{G} 13}=1,3025 \\
\mathrm{P}_{\mathrm{G} 14}=\mathbf{1}, \mathbf{4 0 9 9}\end{array}$ \\
\hline
\end{tabular}

30 barras $\quad 25,27,28,29,30$

$P_{\mathrm{G} 25}=0,5838$

$P_{\mathrm{G} 27}=-0,3439$

$P_{\text {G28 }}=0,7756$

$P_{\mathrm{G} 29}=0,1163$

$\mathrm{P}_{\mathrm{G} 30}=0,1459$

Sul Sudeste

$6,8,63,64$

$\mathrm{P}_{\mathrm{G} 6}=1,7958$

$$
\begin{aligned}
& P_{\mathrm{G} 8}=0,6607 \\
& P_{\mathrm{G} 63}=2,5088 \\
& P_{\mathrm{G} 64}=1,0635
\end{aligned}
$$

Fonte: Autores. 
Tabela 6 - Potências reativas geradas nas barras críticas

\begin{tabular}{|c|c|c|}
\hline Sistemas & Barras críticas & $\begin{array}{l}\text { Potências reativas nas barras } \\
\text { críticas (p.u. na base de } 100 \text { MVA) }\end{array}$ \\
\hline 14 barras & $4,6,7,9,10,12,13,14$ & $\begin{array}{l}Q_{\mathrm{G} 4}=0,5047 \\
Q_{\mathrm{G} 6}=-0,2004 \\
Q_{\mathrm{G} 7}=-0,2569 \\
Q_{\mathrm{G} 9}=0,2268 \\
Q_{\mathrm{G} 10}=1,0989 \\
Q_{\mathrm{G} 12}=0,0736 \\
Q_{\mathrm{G} 13}=2,2957 \\
Q_{\mathrm{G} 14}=1,3763\end{array}$ \\
\hline 30 barras & $25,27,28,29,30$ & $\begin{array}{l}\mathrm{Q}_{\mathrm{G} 25}=1,4587 \\
\mathrm{Q}_{\mathrm{G} 27}=\mathbf{0 , 0 5 7 6} \\
\mathrm{Q}_{\mathrm{G} 28}=-1,5025 \\
\mathrm{Q}_{\mathrm{G} 29}=\mathbf{1}, 7457 \\
\mathrm{Q}_{\mathrm{G} 30}=0,3647\end{array}$ \\
\hline Sul Sudeste & $6,8,63,64$ & $\begin{array}{c}\mathrm{Q}_{\mathrm{G} 6}=0,4902 \\
\mathbf{Q}_{\mathrm{G} 8}=\mathbf{0 , 0 5 3 0} \\
\mathrm{Q}_{\mathrm{G} 63}=-0,6442 \\
\mathbf{Q}_{\mathrm{G} 64}=-\mathbf{0 , 3 7 1 2}\end{array}$ \\
\hline
\end{tabular}

Fonte: Autores.

O maior aumento percentual de margem de carga no sistema com 14 barramentos se deve ao fato de que a área crítica, formada pela barra crítica e as suas duas primeiras vizinhanças, corresponde a $57 \%$ da quantidade de barramentos deste sistema. 
Embora o aumento de margem percentual seja menor nos sistemas com 30 barramentos e no sistema sul sudeste, a quantidade de potência de carga que é atendida, em valores reais, é maior quando comparado ao sistema de 14 barramentos.

\title{
5 CONCLUSÃO
}

Com a elaboração deste trabalho, foi possível realizar o cálculo da capacidade de hospedagem do sistema elétrico de potência utilizando o algoritmo genético.

Com essa implementação, notou-se um incremento significante na margem de carregamento em dois sistemas-teste do IEEE e um sistema real brasileiro. Este resultado proporciona uma solução vantajosa em caso de necessidade de uma ampliação da rede elétrica, evitando condições operativas indesejadas se, por ventura, o limite da capacidade de hospedagem for violado.

Como trabalhos futuros, sugere-se utilizar esta metodologia com outras técnicas de aumento de margem da curva PV conhecidas na literatura.

\section{AGRADECIMENTOS}

Os autores agradecem ao setor de Pesquisa e Extensão do IFMG-Campus Formiga pelo suporte financeiro.

\section{STUDIES ON DISTRIBUTED GENERATION HOSTING CAPACITY IN ELECTRICAL POWER SYSTEMS USING GENETIC ALGORITHM}

\begin{abstract}
There is a growing interest in inserting sources of renewable energy into power systems. For this, it is necessary to know the maximum power that can be installed in a system that does not cause operational problems. This maximum power is defined as the distributed generation hosting capacity of the system. In order to analyze the most appropriate places to insert these sources, the tangent vector technique will be used, while the power values will be calculated through the implementation of a genetic algorithm, which will search the best solutions, within a set, by means of evolutionary techniques. In order to analyze if an individual is better conditioned than the other, the Active Power versus Voltage curve (PV curve), responsible for indicating the maximum charge of an electric system, is used as an objective function. The methodology will be tested on two IEEE (Institute of Electrical and Electronics Engineers) test systems and a real system. In all simulations the operating limits of the analyzed systems will be considered.
\end{abstract}


MOURA, R. S. et al. Estudos sobre a capacidade de hospedagem de geração distribuída em sistemas 14 elétricos de potência utilizando algoritmo genético

Keywords: Electrical system. Accommodation capacity. Genetic algorithm. PV curve.

\section{REFERÊNCIAS}

AFFONSO, Carolina de M.; DA SILVA, Luciano J.; DA SILVA, Luis C.P. Avaliação da estabilidade de tensão do sistema elétrico brasileiro: um caso de estudo. Revista Controle \& Automação, Campinas, v. 19, n. 3, p. 327-336, 2008.

ALMEIDA, Adriano Batista et al. Voltage stability reliability. In: IET Conference on Renewable Power Generation (RPG 2011), 2011, Edinburgh, UK. Disponível em: https://ieeexplore.ieee.org/document/6136170. Acesso em: 19 abr. 2019

ALMEIDA, Adriano Batista et al. Probabilistic voltage stability assessmente considering renewable sources with help of PV and QV curves. IET Renewable Power Generation, Stevenage, UK, v. 7, p. 521-530, 2013.

ALVES, Dilson Amâncio. Obtenção do ponto de máximo carregamento em Sistemas Elétricos de Potência Utilizando Novas Técnicas de Parametrização para o Método de Continuação. 2000. 116 f. Tese (Doutorado em Engenharia Elétrica) - Faculdade de Engenharia Eletrica e de Computação, Universidade Estadual de Campinas, Campinas, 2000.

CANOSSA, Jâine Henrique. Um programa Interativo para estudos de Fluxo de Potência. 2007. 169 f. Dissertação (Mestrado em Engenharia Elétrica) - Programa de Pós-Graduação em Engenharia Elétrica, Universidade Estadual Paulista, São Paulo, 2007.

DE OLIVEIRA, Tiago Elias Castelo. Estudo da Capacidade de Hospedagem de Fontes de Geração Distribuída no Sistema Elétrico de um Campus Universitário. 2015. 125 f.

Dissertação (Mestrado em Engenharia Elétrica) - Programa de Pós-Gradução em Engenharia Elétrica, Universidade Federal de Itajubá, Itajubá, 2015.

FERREIRA, Carlos Aparecido. Novo modelo de transformador com tap variável em regime permanente. 2011. 41 f. Tese (Doutorado em Engenharia Elétrica) - Programa de Pós-Gradução em Engenharia Elétrica, Pontifícia Universidade Católica do Rio de Janeiro, Rio de Janeiro, 2011.

GUIMARÃES, Pablo et al. QV and PV curves as a planning tool of analysis. In: 4 th International Conference on Electric Utility Deregulation and Restructuring and Power Technologies, 2011, Weihai, Shandong, China. Disponível em:

https://ieeexplore.ieee.org/document/5994153. Acesso em: 19 abr. 2019.

KUDUR, Prabha et al. Definition and classification of power system stability IEEE/CIGRE joint task force on stability terms and definitions. IEEE Transactions on Power Systems, Washington DC, USA, v. 19, n. 2, p. 1387-1401, 2004. 
MOURA, R. S. et al. Estudos sobre a capacidade de hospedagem de geração distribuída em sistemas 15 elétricos de potência utilizando algoritmo genético

KAGAN, Nelson et al. Métodos de otimização aplicados a sistemas elétricos de potência. São Paulo: Blucher, 2009.

LINDEN, Ricardo. Algoritmos Genéticos. 3. ed. Rio de Janeiro: Ciência Moderna, 2012.

LODDI, Tarcísio. Cálculo de fluxo de potência unificado em sistemas de transmissão e redes de distribuição através do método de Newton desacoplado rápido com rotação de eixos. 2010. 117 f. Dissertação (Mestrado em Engenharia Elétrica) - Progrmama de PósGraduação em Engenharia Elétrica, Universidade Federal do Paraná, Curitiba, 2010.

MONTICELLI, Alcir José. Fluxo de carga em redes de energia elétrica. São Paulo: Edgard Blücher Ltda. 1983.

MOURA, Renan Souza. Estudo do efeito do tipo da curva QV em simulações dinâmicas em sistemas elétricos de potência. 2016. 87 f. Tese (Doutrorado em Engenharia Elétrica) Programa de Pós-Graduação em Engenharia Elétrica, Universidade Federal de Itajubá, Itajubá, 2016.

SANTOS, Ivan Nunes; ISSA, Laura Elisa. Calculando a capacidade de hospedagem da rede com vistas à inserção de geração distribuída no tocante às distorções harmônicas. In: Conferência de Estudos em Engenharia Elétrica (XII CEEL), Universidade Federal de Uberlândia - UFU, Uberlândia, 2014. Disponível em:

https://www.peteletricaufu.com/static/ceel/doc/artigos/artigos2014/ceel2014_artigo038_r01.p df. Acesso em: 19 abr. 2019.

SANTOS, Ivan Nunes. Considerations on hosting capacity for harmonic distortions on transmission and distribution systems. Electric Power Systems Research, Elsevier Editora Ltda, Rio de Janeiro, v. 119, p. 199-206, 2015.

SOUZA, A.C.Z.; QUINTANA, V.H. Identification of voltage collapse margins in power systems. Canadian Conference on Electrical and Computer Engineering, 14-17 de setembro de 1993.

\section{DADOS DOS AUTORES}

\section{Renan Souza Moura}

E-mail: renan.moura@ifmg.edu.br

Currículo Lattes: http://lattes.cnpq.br/6981517353494441

Doutorado, mestrado e graduação em Engenharia Elétrica pela Universidade Federal de Itajubá (Unifei). Atualmente é professor do Instituto Federal Minas Gerais (IFMG) Campus Formiga. Tem experiência na área de Sistemas Elétricos de Potência nos seguintes temas: análise estática de sistemas elétricos de potência, análise dinâmica de sistemas elétricos de potência, curva QV, Curva PV, transmissão de energia elétrica,geração de energia elétrica e distribuição de energia elétrica. 
MOURA, R. S. et al. Estudos sobre a capacidade de hospedagem de geração distribuída em sistemas 16 elétricos de potência utilizando algoritmo genético

\section{Carine Flávia dos Reis}

E-mail: karyne.f@ hotmail.com

Currículo Lattes: http://lattes.cnpq.br/6579008375021559

Graduanda em Engenharia Elétrica pelo IFMG - Campus Formiga

\section{Rarena Kellen Reis Pedrosa}

E-mail: rarena_pedrosa@yahoo.com.br

Graduação em Engenharia Elétrica pelo IFMG - Campus Formiga

\section{Thuanny Reis Neves}

E-mail: thuannyematheus@hotmail.com

Currículo Lattes: http://lattes.cnpq.br/3819883134948278

Graduação em Engenharia Elétrica pelo IFMG - Campus Formiga 